\title{
The neuropeptide calcitonin gene-related peptide links perineural invasion with lymph node metastasis in oral squamous cell carcinoma
}

Yu Zhang ${ }^{1,2,3,4 \dagger}$, Mingtao Chen ${ }^{1,2,3,4 \dagger}$, Zheqi Liu ${ }^{1,2,3,4}$, Xu Wang ${ }^{1,2,3,4^{*}}$ and Tong $\mathrm{Ji}^{1,2,3,4^{*}}$

\begin{abstract}
Objective: Although perineural invasion (PNI) is well-known to be correlated with and able to predict lymph node metastasis (LNM) in oral squamous cell carcinoma (OSCC), the clinical and molecular correlation between PNI and LNM has not been elucidated, and preoperative biomarkers for LNM prediction in OSCC are urgently needed.

Materials and methods: The correlation between PNI and LNM was retrospectively evaluated using a cohort of 218 patients diagnosed with OSCC. Candidate neuropeptides were screened based on TCGA database and verified via immunohistochemistry and Western blot analyses. ELISA was used to detect calcitonin gene-related peptide (CGRP) in patient plasma. In vitro assays were used to explore the effects of CGRP on OSCC cells.

Results: OSCC patients with PNI had a higher incidence of LNM (69.86\% vs. 26.2\%, $P<0.0001, n=218)$. CGRP expression was upregulated in the PNI niche and in metastatic lymph nodes, and was correlated with poor overall survival of OSCC patients. Preoperative plasma CGRP levels were higher in OSCC patients $(n=70)$ compared to healthy donors $(n=60)$ (48.59 vs. $14.58 \mathrm{pg} / \mathrm{ml}, P<0.0001)$, and were correlated with $\mathrm{LNM}(P<0.0001)$ and $P N I(P=0.0002)$. Preoperative plasma CGRP levels alone yielded an AUC value of 0.8088 to predict LNM, and CGRP levels combined with preoperative T stage reached an AUC value of 0.8590 . CGRP promoted proliferation and migration abilities of OSCC cells, which could be antagonized by either pharmacological or genetic blockade of the CGRP receptor.
\end{abstract}

Conclusions: The neuropeptide CGRP links PNI and LNM in OSCC, and preoperative plasma CGRP levels can be used to predict LNM in OSCC.

Keywords: Oral squamous cell carcinoma, Perineural invasion, Lymph node metastasis, Calcitonin gene-related peptide

*Correspondence: wangx312016@sh9hospital.org.cn; jitong70@sjtu.edu.cn

${ }^{\dagger}$ Yu Zhang and Mingtao Chen contributed equally to this work.

1 Department of Oral Maxillofacial-Head Neck Oncology, Shanghai Ninth

People's Hospital, Shanghai Ninth People's Hospital, Shanghai Jiao Tong

University School of Medicine, Shanghai 200011, China

Full list of author information is available at the end of the article

\section{Introduction}

Oral cancer is a common malignant tumor that occurs in the oral epithelial tissue, and more than $90 \%$ of cases of oral cancer are oral squamous cell carcinoma (OSCC) [1]. One of the most prominent characteristics of OSCC is lymph node metastasis (LNM), even in early-stage disease, which is one of the most significant factors for patient prognosis [2-4]. Many studies suggested that elective neck dissection is recommended 
for early stage OSCC [2]. However, nearly $70 \%$ of early stage OSCC patients undergo unnecessary neck dissection [5]. The sentinel lymph node biopsy has aroused great attention for its considerable sensitivity to detect metastasis in lymph nodes during surgery, thus helping surgeons make personalized operation plan [6,7]. Many researchers now have used molecular biomarkers for LNM prediction. However, most of these biomarkers are based on mRNA or protein expression in tumor tissues [8-10], so are impractical for preoperative prediction. Therefore, preoperative biomarkers for LNM prediction that can be measured non-invasively are urgently needed.

Another important clinicopathological feature of OSCC is perineural invasion (PNI), which has been considered an adverse feature of head and neck cancers in the National Comprehensive Cancer Network guidelines since 2017 [11, 12]. In addition to the clear negative effects of PNI on outcome in OSCC patients [13-15], PNI status has been reported to be correlated with LNM in many studies [16-18]. A study published recently in Cell demonstrated that lymph nodes are innervated by a unique population of sensory nerves [19], indicating a potential anatomical connection between PNI and LNM based on the abundant innervation of the oral cavity. Although some studies found that PNI could be used to predict LNM in OSCC [17], PNI status can only be precisely determined through pathological diagnosis based on tumor tissues, which limits its clinical use. Elucidating the molecular connection between PNI and LNM can thus help to understand their clinical correlation and develop preoperative biomarkers to predict LNM in OSCC patients.

Calcitonin gene-related peptide (CGRP) is often used as a marker of nociceptive nerves and is the most abundant neuropeptide in trigeminal nerves [20,21]. Several studies have reported that CGRP can directly function on various types of cancer like metastatic breast cancer [22], prostate cancer [23] and osteosarcoma [24]. Moreover, endogenous CGRP can promote cancer progression through facilitating tumor-associated angiogenesis [25]. However, the role of CGRP in OSCC and its correlation with PNI and LNM remain unknown.

In the present study, the correlation between PNI and LNM was retrospectively explored using a cohort of 218 patients diagnosed with OSCC. CGRP was screened and verified as the molecular link between PNI and LNM through TCGA IHC and Western blot analyses. The predictive relevance of preoperative plasma CGRP levels was determined through receiver operating characteristic (ROC) analysis. Finally, in vitro assays were used to explore the effects of CGRP on OSCC cells.

\section{Materials and methods} Immunohistochemical analysis

IHC was performed as described previously [26], with primary antibodies against CGRP (C9487, Sigmaaldrcich, America) and PGP9.5 (EPR4118, Abcam, America). Paraffin-embedded $3 \mu \mathrm{m}$-thick sections were deparaffinized, rehydrated and heated with citric acid buffer at $95^{\circ} \mathrm{C}$ for $20 \mathrm{~min}$ for antigen retrieval. Sections were cooled and immersed in $0.3 \%$ hydrogen peroxide for $20 \mathrm{~min}$ to block endogenous peroxidase activity, rinsed in phosphate-buffered saline (PBS) for $5 \mathrm{~min}$ and blocked with $3 \%$ bovine serum albumin (BSA) at room temperature for $20 \mathrm{~min}$. Tissues were incubated with the indicated primary antibodies in a humidified chamber overnight at $4{ }^{\circ} \mathrm{C}$. After several washes with PBS, the sections were incubated with horseradish peroxidase (HRP)-labeled goat anti-mouse or goat ant-rabbit secondary antibody (Gene Tech; Shanghai, China) for $45 \mathrm{~min}$ at $37^{\circ} \mathrm{C}$. Diaminobenzene was used as the chromogen, and hematoxylin was used to counter stain nuclei. The sections were dehydrated, cleared and mounted. The staining intensity was classified into four categories: none (0), weak brown (1), moderate brown (2), and strong brown (3). The proportion of positive cells was determined by the analysis of image J (Rawak Software Inc., Stuttgart, Germany), and the positive aeras of staining was divided into five categories: none (0), $1-25 \%$ (1), $26-50 \%$ (2), $51-75 \%$ (3), $76-100 \%$ (4). The staining score was calculated by multiplying both scores. Staining scores below the mean value were considered to be "low expression," and scores above the mean value were considered to be "high expression."

\section{Patient samples}

Preoperative plasma from 70 OSCC patients and 60 matched healthy donors was collected at the Department of Oral and Maxillofacial Surgery-Head and Neck Oncology, Shanghai Ninth People's Hospital, Shanghai Jiao Tong University School of Medicine. None of the patients had received chemotherapy or radiation therapy prior to surgery. Fresh venous blood samples were collected from fasting subjects, stored in ethylene diamine tetraacetic acid tubes, and processed within $2 \mathrm{~h}$. Eight OSCC tissues and paired adjacent normal tissues were obtained during surgery, immediately frozen in liquid nitrogen, and stored at $-80^{\circ} \mathrm{C}$ until further analysis. The medical records of 218 OSCC patients were obtained from Shanghai Ninth People's Hospital, Shanghai Jiao Tong University School of Medicine. A total of 84 paraffin-embedded primary specimens were collected at the Department of Oral Pathology of Shanghai Ninth People's Hospital and a total of 74 patients were successfully followed up. TNM 
staging was based on the latest American Joint Committee on Cancer staging criteria [27].

\section{Cell culture}

Human HN6 OSCC cells were kindly provided by the University of Maryland Dental School, USA. CAL27 cells were purchased from the American Type Culture Collection (USA). All cells were maintained in Dulbecco's minimum essential medium (Invitrogen, Carlsbad, CA, USA) supplemented with $10 \%$ fetal bovine serum, 100 units $/ \mathrm{ml}$ penicillin, and $100 \mu \mathrm{g} / \mathrm{ml}$ streptomycin and incubated in a humidified atmosphere with $5 \% \mathrm{CO}_{2}$ at $37^{\circ} \mathrm{C}$.

\section{Western blot}

Cells were harvested in RIPA lysis buffer (Beyotime, Haimen, China), and cell lysates were electrophoresed on $4-20 \%$ polyacrylamide gels and transferred to a polyvinylidenedifluoride (PVDF) membrane. Antibodies against the following proteins were used: CGRP (\#14959, CST, USA), CLR (A8533, abclonal, USA) and GAPDH (\#2118, CST, USA). The membranes were incubated with the appropriate primary antibody overnight at $4{ }^{\circ} \mathrm{C}$ and incubated with the appropriate secondary antibody (\#7074, CST, USA) for $1 \mathrm{~h}$ at room temperature.

\section{ELISA assay}

Fresh venous blood samples were drawn into ethylene diamine tetraacetic acid (EDTA) tubes from fasting subjects and processed within $2 \mathrm{~h}$. The whole blood was centrifuged at 3000 round per minute for $15 \mathrm{~min}$, and the supernatant was plasma and was extracted. The human plasma CGRP content is analyzed by human CGRP1ELISA kits (E-EL-H0619c, Elabscience, Wuhan, China) according to manufacturer's introduction.

\section{Clone formation and wound healing assays}

These assays are well described in our previous research [28]. HN6 and Cal27 cells transfected with siCLR or negative control (siNC) for $24 \mathrm{~h}$ were seeded in six-well plates (1000 cells per plate) for 10 days. The cells were washed with PBS, fixed with $4 \%$ paraformaldehyde for $30 \mathrm{~min}$, and stained with Coomassie brilliant blue for $30 \mathrm{~min}$. Colonies with over 50 cells were identified as clones. For wound healing assay, 100\% density of cells were cultured in six-well plates with serum-free medium, and cell monolayer was subsequently scratched with a 200-ul pipette tip. Representative images of cell migration were captured at $0 \mathrm{~h}, 12 \mathrm{~h}$ and $36 \mathrm{~h}$ after injury.

\section{Statistical analysis}

All values are presented as means \pm standard error of the mean (s.e.m.) in bar graphs and scatterplot diagrams. For box plots, center lines represent the median, limits represent the quartiles, and whiskers represent the minimum and maximum values. An unpaired Student's $t$-test with no assumption of equal variance was used for comparisons between two groups. For comparisons of more than two groups, ANOVA (using a general linear model) was used. When the overall $\mathrm{F}$ test was significant $(P<0.05)$, post hoc comparisons using Tukey's method of adjustment were used to assess the presence of any significant pairwise differences. Prognosis prediction performance was evaluated by the area under the ROC curve (AUC). The Youden index was calculated for the prognosis models. Overall survival was calculated using the Kaplan-Meier method. Univariate and multivariate survival analyses were conducted with a Cox regression model. Analyses were performed using GraphPad Prism 8 software (GraphPad Software Inc., San Diego, CA, USA). A two-sided $P$-value of $<0.05$ was considered to indicate statistical significance.

\section{Results}

PNI was correlated with and predicted LNM in OSCC patients without taking $T$ stage into account

We first retrospectively explored the correlation between PNI and the LNM status through a cohort of 218 OSCC patients diagnosed between 2014 and 2020 from Shanghai Ninth People's hospital (Table 1). Patients with PNI

Table 1 Patient Demographics

\begin{tabular}{ll}
\hline Variable & No.(\%) \\
\hline Age & \\
$\leq 60$ & $116(53.2 \%)$ \\
$>60$ & $102(46.8 \%)$ \\
Sex & \\
Male & $143(65.5 \%)$ \\
Female & $75(34.5 \%)$ \\
T stage & \\
T1/T2 & $109(50.0 \%)$ \\
T3/T4 & $109(50.0 \%)$ \\
N stage & \\
LNM (-) & $129(59.2 \%)$ \\
LNM (+) & $89(40.8 \%)$ \\
Tumor location & \\
Tongue & $100(45.9 \%)$ \\
Cheek & $31(14.2 \%)$ \\
Gingiva & $34(15.6 \%)$ \\
Palate & $23(10.6 \%)$ \\
Mouth floor & $17(7.8 \%)$ \\
Mandible and maxilla & $13(5.9 \%)$ \\
Perineural invasion & \\
PNI (-) & $145(66.5 \%)$ \\
PNI (+) & $73(33.5 \%)$ \\
\hline
\end{tabular}



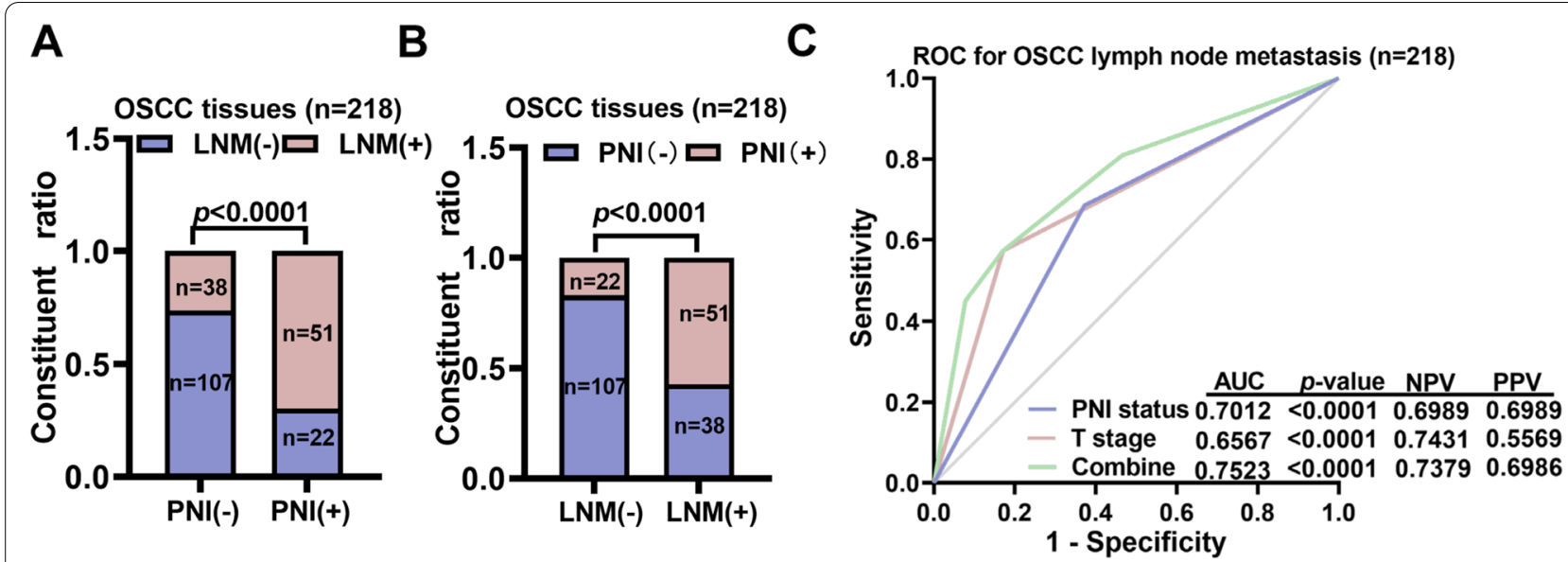

Fig. 1 OSCC patients with PNI had a higher incidence of lymph node metastasis. A OSCC patients with lymph node metastasis (LNM(+)) were classified based on the presence or absence of PNI. B OSCC patients with PNI classified based on the presence or absence of LNM. C The PNI status yielded an AUC value of 0.7012 to predict LNM in OSCC patients, which was better than that obtained for the T stage. Both the PNI status and T stage were included in the multivariant logistic regression model to yield the combination value

had a higher incidence of LNM (51/73 [69.86\%] vs. 38/145 [26.2\%], $P<0.0001$ ) (Fig. 1A). Patients with LNM had a high incidence of PNI (51/89 [57.3\%] vs. 17.05\%, $P<0.0001$ ) (Fig. 1B). We subsequently determined if PNI status could be used to predict LNM. By ROC analysis, we found that PNI status yielded an AUC value of 0.7012 to predict LNM in OSCC patients, which was better than that obtained for the $\mathrm{T}$ stage. The sensitivity was $57.3 \%$ and the specificity was $82.95 \%$, which was not considered satisfactory (Fig. 1C).

The neuropeptide CGRP correlated with both PNI and LNM Despite the strong correlation between PNI and LNM, PNI status is usually determined after surgery through histopathological analysis, which limits its application. Due to the abundant innervation of nociceptive nerves branching from the trigeminal nerves in oral mucosa, we postulated that circulating neuropeptides released from nociceptors could potentially predict PNI status preoperatively. By mining the single-cell database of the murine nerve system (http://mousebrain.org/genesearch. html), we found that Calca (encoding CGRP-I) was the most abundant neuropeptide-encoding mRNA in dorsal root ganglia and trigeminal ganglia. We next explored the mRNA expression of several neuropeptides, including Calca, as well as Calcb and Tac1, which are considered to be coexpressed with Calca, in different tumor types according to the TCGA database (Fig. 2A). Of all these candidates, only Calca was correlated with poor overall survival in head and neck cancer (hazard ratio $[\mathrm{HR}]=1.4$, $n=583, P=0.035$ ) (Fig. 2A and S1).

Further, we measured protein levels of CGRP in OSCC tissues. Interestingly, in OSCC patients with PNI, expression of CGRP was higher in tumor tissues compared to adjacent normal tissues, while in patients without PNI, expression of CGRP was lower and similar between tumor tissues and adjacent normal tissues (Fig. 2B). This implied that CGRP could have been secreted from invading nerves.

To test this hypothesis, 84 OSCC tissues were subjected to IHC staining against CGRP and the nerve marker PGP9.5. We found that CGRP was co-expressed with PGP9.5 and showed strong staining at the perineural region (Fig. 2C-D). Quantitative analysis revealed that the OSCC tissues with PNI had a higher CGRP staining score in OSCC tumor tissues $(P=0.0103)$ (Fig. 2E). More

(See figure on next page.)

Fig. 2 The nociceptor marker calcitonin gene-related peptide (CGRP) was correlated with both PNI and LNM in OSCC patients. A Kaplan-

Meier survival analysis of cancer patients stratified by different neuropeptide mRNA levels according to the TCGA database. B Protein

expression of CGRP in tumor tissues and adjacent normal tissues in PNI(-) and PNI(+) OSCC tissues. C Representative IHC images of OSCC tissues with different CGRP staining intensity. D Representative IHC images of PNI tissue from OSCC patients stained for CGRP and the nerve marker PGP9.5. E Quantification of CGRP scores in PNI(-) and PNI(+) OSCC tissues. F Representative immunohistochemistry images of a metastatic lymph node stained for CGRP. G CGRP staining levels in LNM(-) and LNM(+) OSCC tissues. BRCA, Breast invasive carcinoma; COAD, Colon adenocarcinoma; ESCA, Esophageal carcinoma; GBM, Glioblastoma multiforme; HNSCC, Head and Neck squamous cell carcinoma; LGG, Brain Lower Grade Glioma; LUSC, Lung squamouscell carcinoma 
A

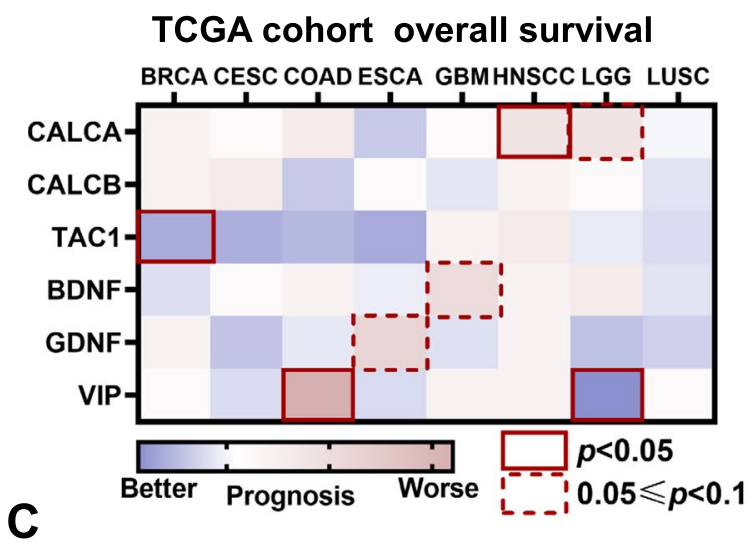

B

PNI(+) OSCC tumor tissues
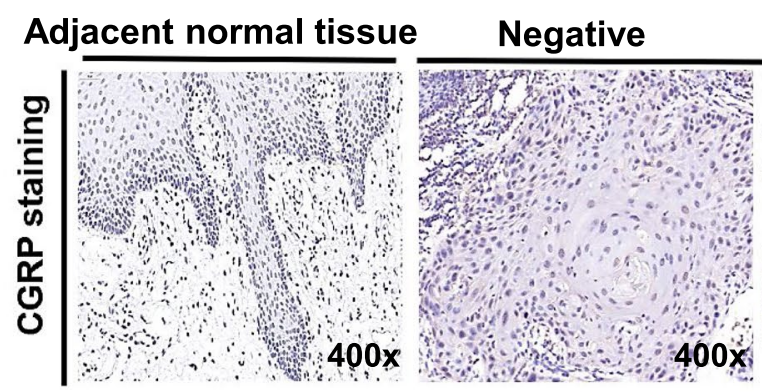

Moderate

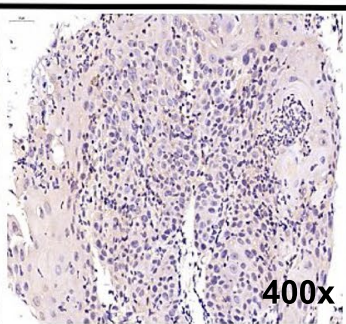

Strong

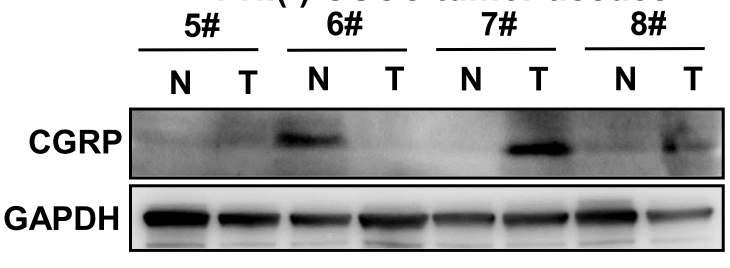

PNI(-) OSCC tumor tissues

D

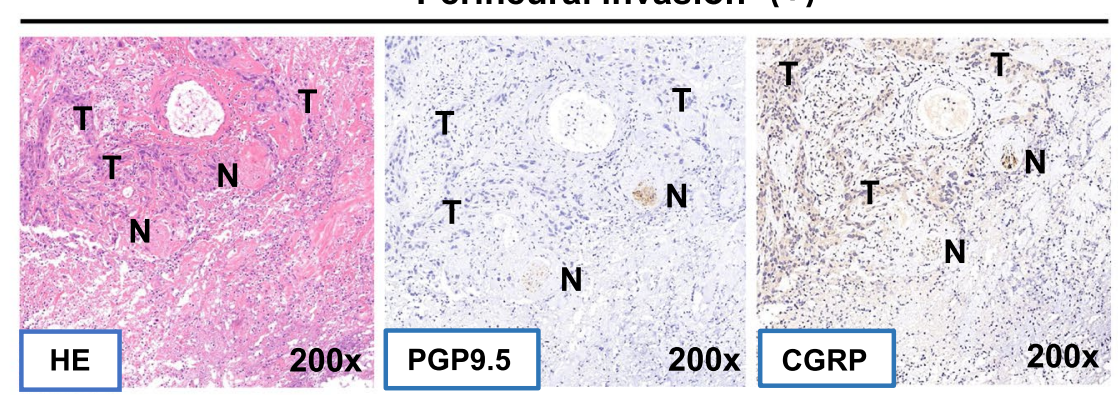

$\mathbf{F}$

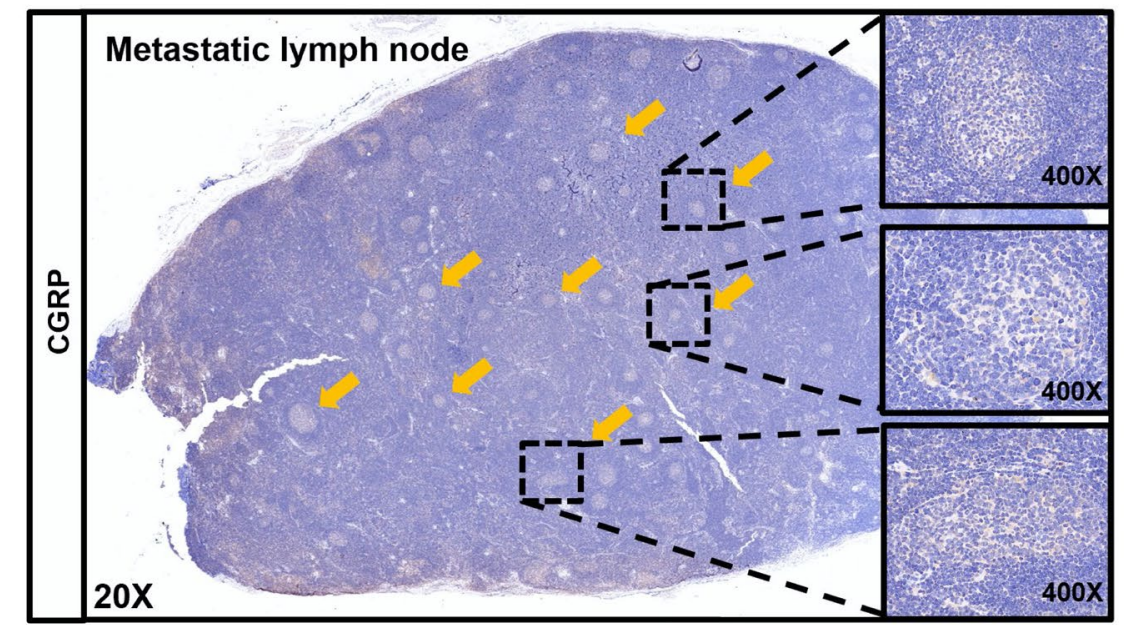

E Perineural Invasion

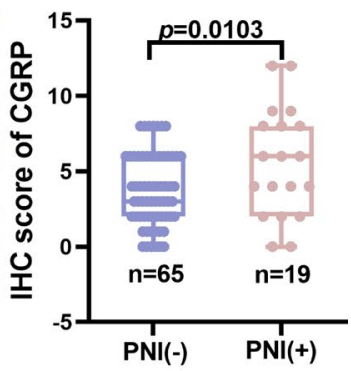

G

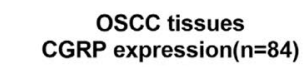

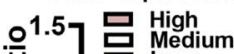

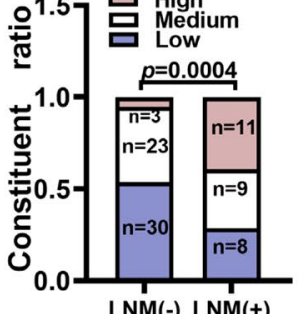

Fig. 2 (See legend on previous page.) 
A

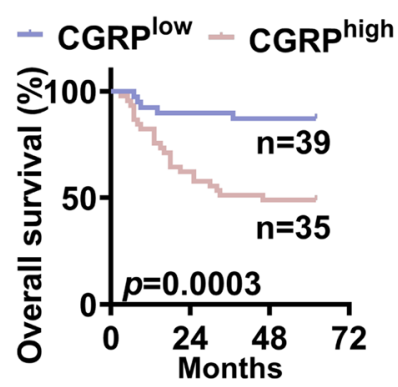

C Cox multivariate regression for overall survival

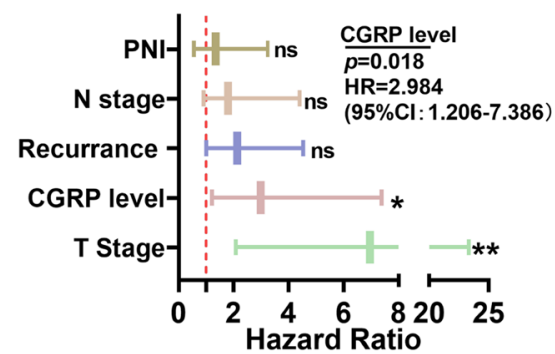

B

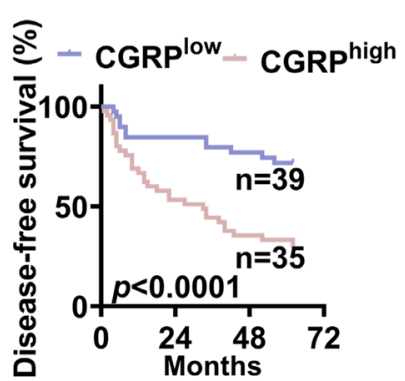

D

Cox multivariate regression for disease free survival

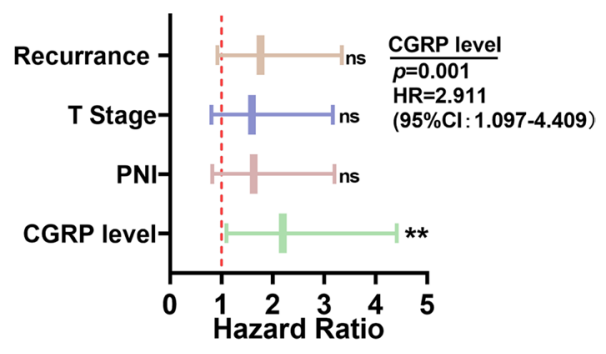

Fig. 3 High CGRP expression in OSCC tumor tissues was correlated with poor overall survival. A-B Kaplan-Meier analysis revealed that OSCC patients with high CGRP expression in tumor tissues had worse overall survival rate and disease-free survival rate. C Multivariate Cox regression analysis revealed CGRPexpression in OSCC tumor tissues is an independent risk factor for overall survival in OSCC patients. D Multivariate Cox regression analysis revealed CGRP expression in OSCC tumor tissues was an independent risk factor for disease-free survival in OSCC patients
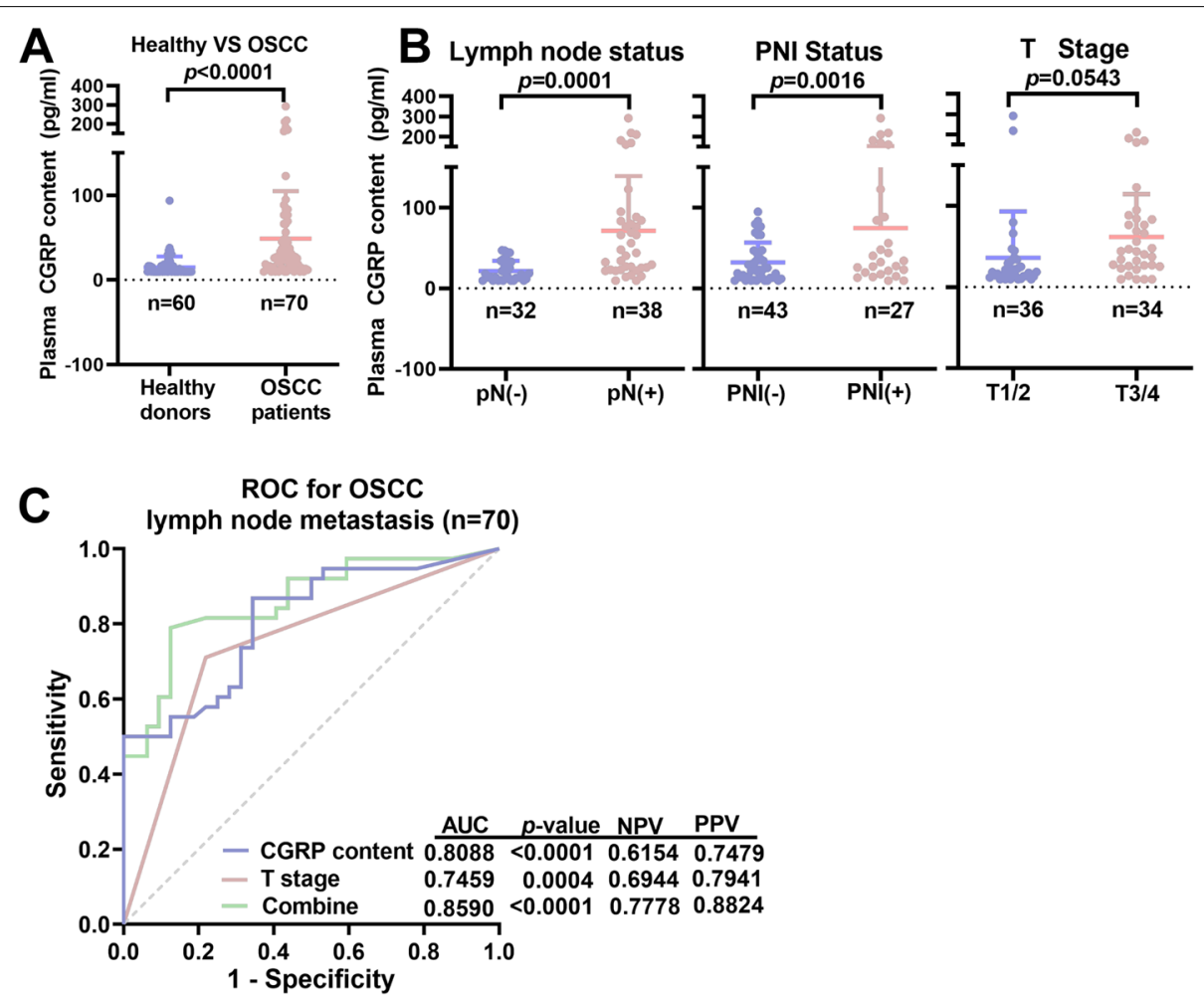

Fig. 4 Circulating CGRP levels were predictive of LNM in OSCC patients. A Plasma CGRP levels in OSCC patients $(n=70)$ and healthy donors $(n=60)$, as analyzed by ELISA. B High CGRP levels were correlated with LNM, PNI, but not advanced T stage(T3/T4) in OSCC patients. C The combination of preoperative Tstage and plasma CGRP levels was calculated by multivariant logistic regression and yielded an AUC value of 0.8590 to predict LNM in OSCC patients 
importantly, we found that CGRP was stained in the metastatic niches of lymph nodes (Fig. 2F). Consistent with this, the quantitative analysis identified that OSCC tissues with LNM had higher CGRP expression in OSCC tumor tissues $(P=0.0004)$ (Fig. 2G).

Furthermore, expression of CGRP in OSCC tumor tissues was an independent risk factor of both overall survival and disease-free survival rate in OSCC patients (Fig. 3A-D).

\section{Preoperative plasma CGRP levels predicted LNM in OSCC patients}

We found that CGRP expression in tumor tissues and metastatic lymph nodes was correlated with both PNI and LNM in OSCC patients, and that trigeminal ganglion neurons were pseudounipolar. Therefore, we postulated that substances with biological activity could be released in peripheral nerve endings once the nociceptive nerves are activated, and explored the feasibility of using peripheral CGRP as a preoperative marker to predict LNM. Peripheral blood from 60 healthy donors and 70 age- and sex-matched OSCC patients was collected, and plasma was extracted to measure CGRP levels by
ELISA. Strikingly, CGRP levels were significantly higher in OSCC patients compared to healthy controls (48.59 vs. $14.98 \mathrm{pg} / \mathrm{ml}, P<0.0001$ ) (Fig. $4 \mathrm{~A}$ ). Among the 70 OSCC patients, CGRP levels were significantly higher in patients with LNM $(P<0.0001)$ and PNI $(P=0.0016)$, but not correlated with T stage $(P=0.0543)$ (Fig. 4B).

We then explored the predictive efficiency of preoperative plasma CGRP levels. ROC analysis revealed that CGRP alone yielded an AUC value of 0.8088 , with a negative predictive value of $61.54 \%$ and a positive predictive value of $74.79 \%$ (Fig. 4C). The best cutoff value of CGRP levels was $21.66 \mathrm{pg} / \mathrm{ml}$, which yielded a Youden's index of 0.5247. Preoperative $\mathrm{T}$ stage was easy to determine based on clinical features and preoperative imaging. We found that the combination of CGRP and preoperative T stage yielded an AUC value of $0.8590(P<0.0001)$, with a negative predictive value of $77.78 \%$ and a positive predictive value of $88.24 \%$ (Fig. 4 C).

Taken together, these findings demonstrated that preoperative plasma CGRP levels were correlated with LNM and PNI, and could therefore be used to predict LNM in OSCC.

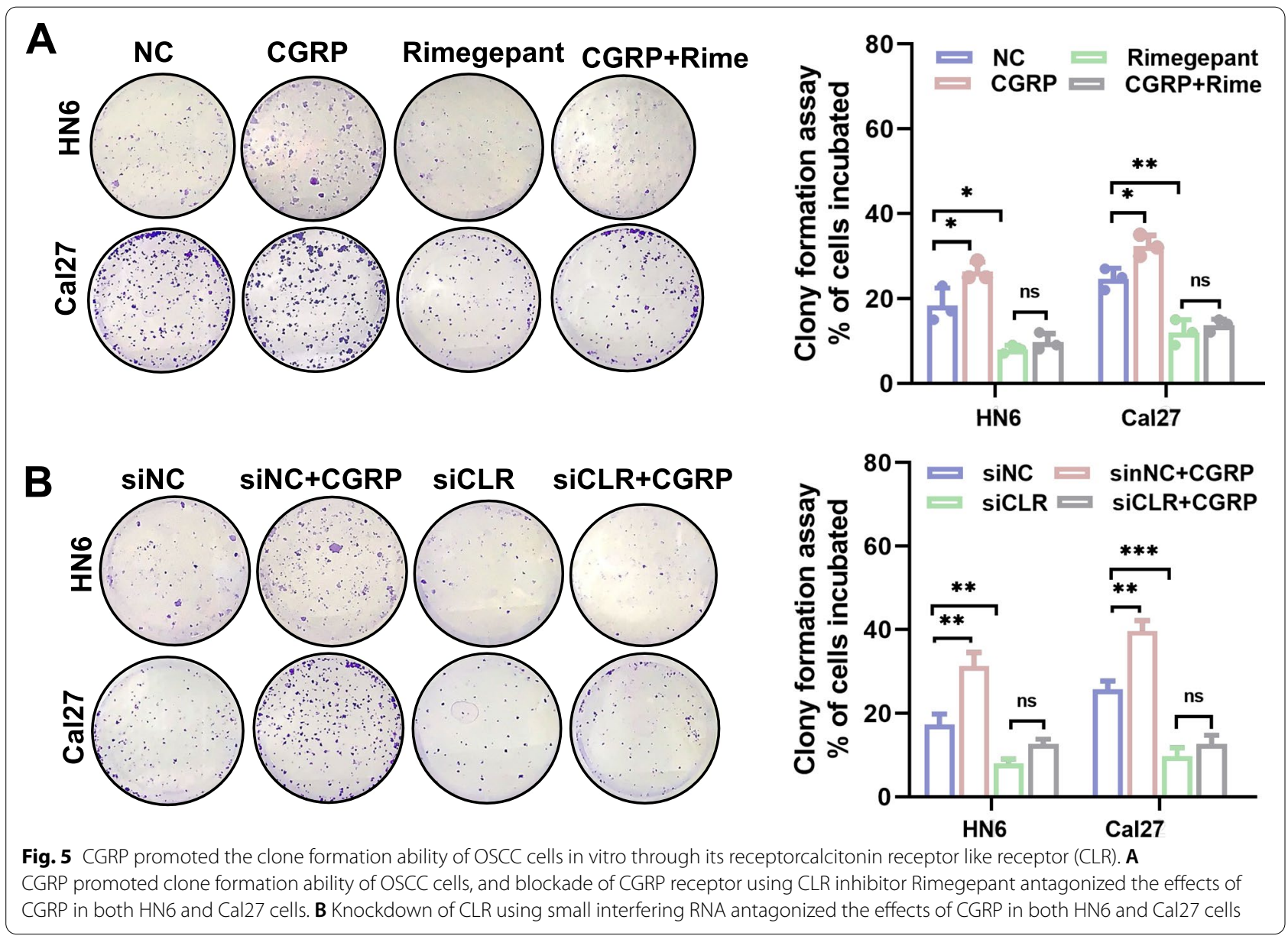


CGRP promoted differentiation of OSCC cells into malignant phenotypes in vitro through its receptor CLR

Because CGRP was correlated with LNM and PNI in OSCC, we next investigated whether CGRP could influence the phenotypes of OSCC cells. Colony formation and migration are two essential functions for cancer cell metastasis. CGRP increased colony formation ability (Fig. 5A, B) and wound healing ability (Fig. 6A, B) of the human OSCC cell lines HN6 and Cal27. CGRP needs to bind with its receptor CLR (encoded by $C A L C R L$ ) on cell membrane and transmit downstream signals. These promoting effects of CGRP could be antagonized by either treatment with the CGRP receptor antagonist Rimegepant or knockdown of the CGRP receptor CLR using small interfering RNA (siRNA) (Fig. 5A-B, 6A-B and S2).

\section{Discussion}

In the present study, we found that PNI was correlated with LNM in OSCC. We further identified CGRP as a molecular link between PNI and LNM. Most importantly, preoperative plasma CGRP levels, combined with preoperative pain status and $\mathrm{T}$ stage, can be used to predict LNM.
As we found that OSCC patients with PNI had a higher incidence of LNM, several studies have reported that the PNI status was correlated with LNM in OSCC [2931] . But the underlying biological connection between PNI and LNM is rarely reported. Nerves, as an important component in tumor microenvironment, has now emerged as a promising candidate [32, 33]. The oral cavity is densely innervated by the sensory nerves branching from the trigeminal nerves [34]. Prior studies have demonstrated the role of nerves in OSCC development. For example, Myers et al. reported that p53 loss led to the reprogramming of sensory nerves into sympathetic nerves, which promoted tumor progression [35]. A recent study demonstrated that lymph nodes are innervated by a unique population of sensory nerves [19]. These studies together indicate that nerves could connect different organs such as the oral mucosa and lymph nodes. If so, there may exist an anatomical and molecular connection between PNI and LNM in OSCC. Further studies should use in vivo models to verify the correlation of PNI and LNM via direct regulation of nerves.

Based on the clinical correlation between PNI and LNM, many researchers found that PNI can well predict the LNM [36]. However, the PNI status can be only determined after surgical dissection of tumor tissue,

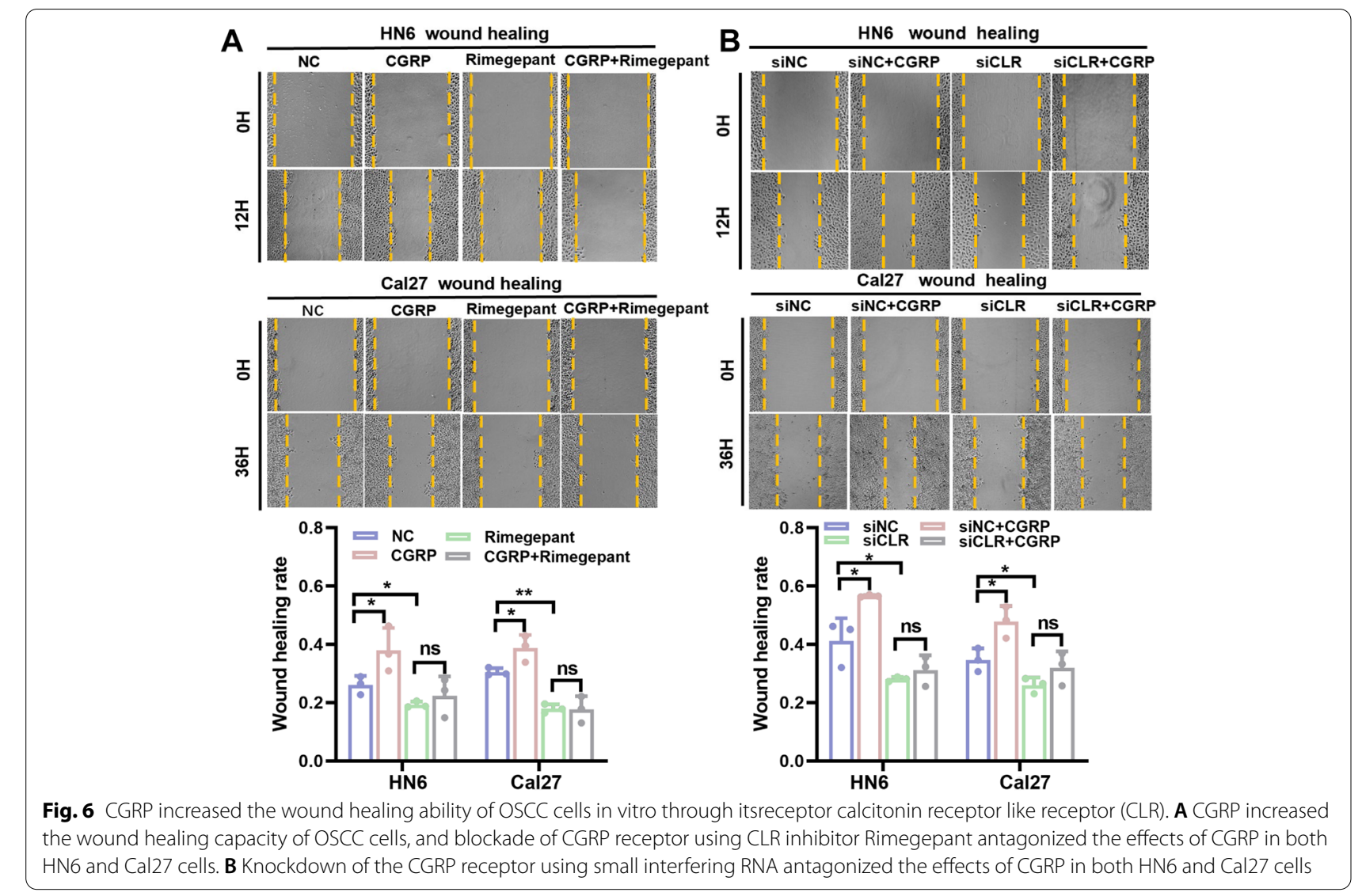


limiting its practical use in LNM prediction, and the preoperative non-invasive examinations are more attractive to clinicians to make personalized treatment. Preoperative blood tests are promising tools to predict survival and LNM in patients with malignant tumors such as colorectal cancer [37], renal cell cancer [38] and OSCC [39]. We found that preoperative plasma CGRP levels were predictive of LNM, yielding an AUC value of 0.8088 . More importantly, the combination of preoperative plasma CGRP levels and preoperative $T$ stage yielded an AUC value of 0.8590 , providing a promising predictive model. It can partly be explained by that tumors with advanced $\mathrm{T}$ stages are not always companied with PNI. However, the correlation between preoperative plasma content of CGRP and the prognosis is unknown. Longterm follow-up is still required to answer this question.

CGRP, which plays a key role in migraine, has been reported to promote tumor progression by either directly affecting tumor cells [40] or promoting tumor-associated angiogenesis [25, 41]. More importantly, we found that the CGRP expression in OSCC tumor tissues was higher in OSCC patients with PNI or LNM and CGRP was found to promote the clone formation and wound healing abilities of OSCC cells. Due to that CGRP is often used as a marker of nociceptive nerves and is the most abundant neuropeptide in trigeminal nerves $[20,21]$, we think that the neurogenic CGRP may enhance the malignant phenotypes of cancer cell and lead to PNI and LNM. Further studies may use genetic modification mice to specifically regulate CGRP in nerves to verify this hypothesis.

\section{Conclusions}

PNI and LNM are correlated in OSCC. The neuropeptide CGRP linked PNI and LNM in OSCC, and preoperative plasma CGRP levels could be used to predict LNM in OSCC patients.

\section{Supplementary Information}

The online version contains supplementary material available at https://doi. org/10.1186/s12885-021-08998-9.

\section{Additional file 1.}

\section{Acknowledgements}

Not applicable.

\section{Authors' contributions}

YZ and TJ conceptualized the project; $Y Z$ and ZQL conducted experiments; $Y Z$ and MTC performed data analysis; $Y Z$ wrote the manuscript, and $X W$ reviewed and edited the manuscript. TJ and XW acquired funding for the project. All authors have read and agreed to the published version of the manuscript.

\section{Funding}

This study was supported by the National Natural Science Foundation of China (Grant No. 81872189 and 82173147 to Tong Ji, Grant No.82073384 to Xu
Wang),Shenkang Development Center (Grant No. SHDC2020CR6010) to Tong $\mathrm{Ji}$ and Biobank Program of Shanghai Ninth People's Hospital (YBKB201908) to Tong Ji

\section{Availability of data and materials}

All data generated or analyzed during this study are included in this published article.

\section{Declarations}

\section{Ethics approval and consent to participate}

This study was approved by the Ethics Committee of the Ninth People's Hospital, Shanghai Jiao Tong University School of Medicine (the certificate number: SH9H-TK128-1). All methods were carried out in accordance with relevant guidelines and regulations. The written informed consents were obtained from all subjects included in this study.

\section{Consent for publication}

Not applicable.

\section{Competing interests}

The authors declare no conflict of interest.

\section{Author details}

'Department of Oral Maxillofacial-Head Neck Oncology, Shanghai Ninth People's Hospital, Shanghai Ninth People's Hospital, Shanghai Jiao Tong University School of Medicine, Shanghai 200011, China. ${ }^{2}$ College of Stomatology, Shanghai Jiao Tong University, Shanghai 200011, China. ${ }^{3}$ National Center for Stomatology, National Clinical Research Center for Oral Diseases, Shanghai 200011,

China. ${ }^{4}$ Shanghai Key Laboratory of Stomatology, Shanghai 200011, China.

Received: 10 June 2021 Accepted: 10 November 2021

Published online: 20 November 2021

\section{References}

1. Chow L. Head and neck Cancer. N Engl J Med. 2020;382(1):60-72. https:// doi.org/10.1056/NEJMra1715715.

2. D'Cruz A, Vaish R, Kapre N, Dandekar M, Gupta S, Hawaldar R, et al. Elective versus therapeutic neck dissection in node-negative Oral Cancer. N Engl J Med. 2015;373(6):521-9. https://doi.org/10.1056/NEJMoa1506 007.

3. Ren Z, Xu J, Li B, Fan T, Ji T, Zhang C. Elective versus therapeutic neck dissection in node-negative oral cancer: evidence from five randomized controlled trials. Oral Oncol. 2015;51(11):976-81. https://doi.org/10. 1016/j.oraloncology.2015.08.009.

4. D'Cruz A, Dandekar M. Elective versus therapeutic neck dissection in the clinically node negative neck in early oral cavity cancers: do we have the answer yet? Oral Oncol. 2011;47(9):780-2. https://doi.org/10. 1016/j.oraloncology.2011.06.013.

5. Liu H, Wang S, Cheng A, Han Z, Feng Z, Guo C. GPD1L is negatively associated with HIF1a expression and predicts lymph node metastasis in oral and HPV- Oropharyngeal cancer. Oral Dis. 2020. https://doi.org/ 10.1111/odi.13694.

6. Nieuwenhuis E, Kolenaar B, van Bemmel A, Hof J, van Baarlen J, Christenhusz A, et al. A complete magnetic sentinel lymph node biopsy procedure in oral cancer patients: a pilot study. Oral Oncol. 2021;121:105464. https://doi.org/10.1016/j.oraloncology.2021.105464.

7. Kim J, Ku M, Yang J, Byeon H. Recent developments of ICG-guided sentinel lymph node mapping in Oral Cancer. Diagnostics (Basel, Switzerland). 2021;11(5). https://doi.org/10.3390/diagnostics 1050891.

8. De Herdt M, van der Steen B, van der Toom Q, Aaboubout Y, Willems S, Wieringa $\mathrm{M}$, et al. The potential of MET Immunoreactivity for prediction of lymph node metastasis in early Oral tongue squamous cell carcinoma. Front Oncol. 2021;11:638048. https://doi.org/10.3389/fonc.2021. 638048.

9. Wang S, Li T, Liu H, Wei W, Yang Y, Wang C, et al. A combined prediction model for lymph node metastasis based on a molecular panel and 
Clinicopathological factors in Oral squamous cell carcinoma. Front Oncol. 2021;11:660615. https://doi.org/10.3389/fonc.2021.660615.

10. Qiao B, Zhao M, Wu J, Wu H, Zhao Y, Meng F, et al. A novel RNA-Seqbased model for preoperative prediction of lymph node metastasis in Oral squamous cell carcinoma. Biomed Res Int. 2020;2020:4252580. https://doi.org/10.1155/2020/4252580.

11. Colevas A, Yom S, Pfister D, Spencer S, Adelstein D, Adkins D, et al. NCCN guidelines insights: head and neck cancers, version 1.2018. J Natl Compr Cancer Netw. 2018;16(5):479-90. https://doi.org/10.6004/ jnccn.2018.0026.

12. Adelstein D, Gillison M, Pfister D, Spencer S, Adkins D, Brizel D, et al. NCCN guidelines insights: head and neck cancers, version 2.2017. J Natl Compr Cancer Netw. 2017;15(6):761-70. https://doi.org/10.6004/ jncen.2017.0101.

13. Park J, Megow A, Swalling A, Hodge J, Foreman A, Boase S, et al. Prognosis of oral squamous cell carcinoma with perineural invasion: A comparative study of classification types. Clin Otolaryngol. 2020:45(1):99-105. https://doi.org/10.1111/coa.13472.

14. Hasmat S, Ebrahimi A, Gao K, Low T, Palme C, Gupta R, et al. Multifocal perineural invasion is a better prognosticator than depth of invasion in oral squamous cell carcinoma. Head Neck. 2019;41(11):3992-9. https:// doi.org/10.1002/hed.25940.

15. Subramaniam N, Balasubramanian D, Murthy S, Kumar N, Vidhyadharan $\mathrm{S}$, Vijayan S, et al. Predictors of locoregional control in stage I/II oral squamous cell carcinoma classified by AJCC 8th edition. Eur J Surg Oncol. 2019;45(11):2126-30. https://doi.org/10.1016/j.ejso.2019.05.018.

16. Alzahrani S, Feng Z, Cheng A, Han Z, Moe J, Ward B. Is Perineural invasion a reasonable Indicator for neck dissection in CT1NOMO squamous cell carcinoma of the Oral cavity? J Oral Maxillofac Surg. 2021;79(3):704-11. https://doi.org/10.1016/j.joms.2020.08.038.

17. Jin $W$, Zhu M, Zheng $Y, W u Y$, Ding $X, W u H$, et al. Perineural invasion, lactate dehydrogenase, globulin, and serum sodium predicting occult metastasis in oral cancer. Oral Dis. 2020. https://doi.org/10.1111/odi. 13750.

18. Zhu J, Zhou R, Wang Y, Yu M. Perineural invasion as a prognostic factor in head and neck squamous cell carcinoma: a systematic review and meta-analysis. Acta Otolaryngol. 2019;139(11):1038-43. https://doi.org/ 10.1080/00016489.2019.1655167.

19. Huang S, Ziegler CGK, Austin J, Mannoun N, Vukovic M, OrdovasMontanes J, et al. Lymph nodes are innervated by a unique population of sensory neurons with immunomodulatory potential. Cell. 2021;184(2):441-459 e25. https://doi.org/10.1016/j.cell.2020.11.028

20. Eftekhari S, Salvatore C, Calamari A, Kane S, Tajti J, Edvinsson L. Differential distribution of calcitonin gene-related peptide and its receptor components in the human trigeminal ganglion. Neuroscience. 2010;169(2):683-96. https://doi.org/10.1016/j.neuroscience.2010.05. 016.

21. Nguyen $M$, Wu Y, Bonilla $L$, von Buchholtz $L$, Ryba N. Diversity amongst trigeminal neurons revealed by high throughput single cell sequencing. PLoS One. 2017;12(9):e0185543. https://doi.org/10.1371/journal. pone. 0185543 .

22. Gutierrez S, Boada MD. Neuropeptide-induced modulation of carcinogenesis in a metastatic breast cancer cell line (MDA-MB-231). Cancer Cell Int. 2018;18(216). https://doi.org/10.1186/s12935-018-0707-8.

23. Nagakawa O, Ogasawara M, Fujii H, Murakami K, Murata J, Fuse H, et al. Effect of prostatic neuropeptides on invasion and migration of PC-3 prostate cancer cells. Cancer Lett. 1998;133(1):27-33.

24. Drissi H, Lieberherr M, Hott M, Marie PJ, Lasmoles F. Calcitonin generelated peptide (CGRP) increases intracellular free $\mathrm{Ca} 2+$ concentrations but not cyclic AMP formation in CGRP receptor-positive osteosarcoma cells (OHS-4). Cytokine. 1999;11(3):200-7.

25. Toda M, Suzuki T, Hosono K, Hayashi I, Hashiba S, Onuma Y, et al. Neuronal system-dependent facilitation of tumor angiogenesis and tumor growth by calcitonin gene-related peptide. Proc Natl Acad Sci U S A. 2008;105(36):13550-5. https://doi.org/10.1073/pnas.0800767105.
26. Zhang Y, Peng C, Tian Z, Cao W, Yang X, Ji T. Inflammatory myofibroblastic tumor in the head and neck-a neoplasm with both tumor features and inflammation. Oral Surg Oral Med Oral Pathol Oral Radiol. 2020;130(5):e316-23. https://doi.org/10.1016/j.00oo.2020.02.008.

27. Amin MB, Edge S, Greene F, Byrd DR, Brookland RK, Washington MK, et al. AJCC Cancer staging manual. 8th ed. New York: Springer International Publishing; 2017.

28. Lin C, Ren Z, Yang X, Yang R, Chen Y, Liu Z, et al. Nerve growth factor (NGF)-TrkA axis in head and neck squamous cell carcinoma triggers EMT and confers resistance to the EGFR inhibitor erlotinib. Cancer Lett. 2020;472:81-96. https://doi.org/10.1016/j.canlet.2019.12.015.

29. Lee T, Chiu P, Li W, Yang M, Wei P, Chu P, et al. Nerve-tumour interaction enhances the aggressiveness of oral squamous cell carcinoma. Clin Otolaryngol. 2019;44(6):1087-95. https://doi.org/10.1111/coa.13452.

30. Wei P, Li W, Tai S. Discrete Perineural Invasion Focus Number in Quantification for T1-T2 Oral Squamous Cell Carcinoma. Otolaryngol Head Neck Surg. 2019;160(4):635-41. https://doi.org/10.1177/0194599818 808510.

31. Chatzistefanou I, Lubek J, Markou K, Ord R. The role of neck dissection and postoperative adjuvant radiotherapy in $\mathrm{CNO}$ patients with PNI-positive squamous cell carcinoma of the oral cavity. Oral Oncol. 2014;50(8):753-8. https://doi.org/10.1016/j.oraloncology.2014.05.005.

32. Boilly B, Faulkner S, Jobling P, Hondermarck H. Nerve dependence: from regeneration to Cancer. Cancer Cell. 2017;31(3):342-54. https:// doi.org/10.1016/j.ccell.2017.02.005.

33. Saloman JL, Albers KM, Rhim AD, Davis BM. Can stopping nerves, Stop Cancer? Trends Neurosci. 2016;39(12):880-9. https://doi.org/10.1016/j. tins.2016.10.002.

34. Mu L, Sanders I. Human tongue neuroanatomy: nerve supply and motor endplates. Clin Anat. 2010;23(7):777-91. https://doi.org/10. 1002/ca.21011.

35. Amit M, Takahashi H, Dragomir M, Lindemann A, Gleber-Netto F, Pickering $C$, et al. Loss of p53 drives neuron reprogramming in head and neck cancer. Nature. 2020;578(7795):449-54. https://doi.org/10.1038/ s41586-020-1996-3.

36. Yang X, Tian X, Wu K, Liu W, Li S, Zhang Z, et al. Prognostic impact of perineural invasion in early stage oral tongue squamous cell carcinoma: results from a prospective randomized trial. Surg Oncol. 2018;27(2):123-8. https://doi.org/10.1016/j.suronc.2018.02.005.

37. Nassar F, Msheik Z, Nasr R, Temraz S. Methylated circulating tumor DNA as a biomarker for colorectal cancer diagnosis, prognosis, and prediction. Clin Epigenetics. 2021;13(1):111. https://doi.org/10.1186/ s13148-021-01095-5.

38. Schutz F, Xie W, Donskov F, Sircar M, McDermott D, Rini B, et al. The impact of low serum sodium on treatment outcome of targeted therapy in metastatic renal cell carcinoma: results from the international metastatic renal cell Cancer database consortium. Eur Urol. 2014;65(4):723-30. https://doi.org/10.1016/j.eururo.2013.10.013.

39. Morand G, Ikenberg K, Vital D, Cardona I, Moch H, Stoeckli S, et al. Preoperative assessment of CD44-mediated depth of invasion as predictor of occult metastases in early oral squamous cell carcinoma. Head Neck. 2019;41(4):950-8. https://doi.org/10.1002/hed.25532.

40. Angenendt L, Bormann E, Pabst C, Alla V, Gorlich D, Braun L, et al. The neuropeptide receptor calcitonin receptor-like (CALCRL) is a potential therapeutic target in acute myeloid leukemia. Leukemia. 2019. https:// doi.org/10.1038/s41375-019-0505-x.

41. Zhang Y, Lin C, Wang X, Ji T. Calcitonin gene-related peptide: a promising bridge between cancer development and cancer-associated pain in oral squamous cell carcinoma. Oncol Lett. 2020;20(5):253. https:// doi.org/10.3892/ol.2020.12116.

\section{Publisher's Note}

Springer Nature remains neutral with regard to jurisdictional claims in published maps and institutional affiliations. 